\title{
Adaptive Remote Sensing Technique Implementation on Configurable Multibot Platform
}

\author{
Mr. B. Vinoba ${ }^{1}$, Mr. P. Saravanan, M.Tech ${ }^{2}$ \\ ${ }^{1} P G$ Scholar,Electrical\& Electronics Eng., GanadipathyTulsi's Jain Engineering college,Kaniyambadi, \\ Vellore - 632102 \\ ${ }^{2}$ Assistantprofessor,Electrical\& Electronics Eng, GanadipathyTulsi’s Jain Engineering college, \\ Kaniyambadi, Vellore-632102,
}

\begin{abstract}
Swarm robotics is a growing area in the field of mobile robotics, however limited work has been done in the area of applications for swarm robotics. Several applications such as remote environmental sensing, security, and search and rescue are several immediate applications of swarm robotics. However, this field suffers from a lack of complete swarm robot platforms, which includes: a computational platform capable of hosting robust control and communication systems, a communications and data exchange model capable of robust data transfer in a dynamic network, and finally an easy to use and modify human machine interface.hence using the dynamic robot for mining application.also using the sarm intelligence environmental sensing application.therebyadative remote sensing technique are implementated.
\end{abstract}

Index Term: Robotics, Automation,Communication,pc, Wireless Technologies

\section{Introduction}

The use of machines in non-human operable conditions or such conditions which are costly to implement a human solution is not a new development. Recently, the hardware needed to perform such tasks in these conditions has become readily available at an affordable cost. This allows more widespread development for applications that have generally been ignored, such as automated environmental monitoring, security, and search/rescue. Both security and search/rescue applications cover a similar team-basedApproach, where teams of technicians in the form of either a security patrol or search team is deployed to service geographic locations, with servicing in this case, simply inspecting the environment. These team-based methods are costly in terms both time and capital resources, especially when the scenario dictates frequent monitoring of the locations in question. However, the team approached can be mechanized and automated by using simple, swarm robotics techniques. Swarm robotics, is an emerging applications field of mobile robotics, which involves multitudes of simple robots working in conjunction with each other to complete a task which would otherwise be impossible or implausible to complete with a single robot solution. Swarm robotics has been proven in the fields of defense [1] to wireless network creation [2,3] and have major applications in the area practical swarm based algorithms, which are too numerous to list in this work.

A major barrier to the use of swarm robots for the monitoring and research application includes the lack of a complete solution, encompassing all sub-systems (embedded hardware/software, data exchange, and user interface) required, which is both easy to use, and portable amongst various robot architectures used. Several different implementations exist for the remote sensing application [4,5,6],however these suffer from a lack of a flexible solution which can be adjusted based on the application. Therefore, a complete swarm robotic system is proposed, which encompasses the hardware/software to interface to, and control a small robot, a communications and data exchange component to manage information between each robot node, an finally a pc based human/machine interface (HMI) in order to integrate mobile technologies in the system. For the purposes of this design, the remote environmental sensing problem is targeted, however, only the robot itself need be modified to suit other applications.

The rest of this paper is organized as follows. Section II Presents the platform architecture, and identifies the components implemented. The communications and data exchange components which allow information access and connectivity are described in section III. The ismobile robot data interfacedescribed in section IV, while the physical robot platform is described in section V. Finally, the HMI is described in section VI, while results and conclusions are described in sections VII, respectively.

\section{Platform Overview}

The overall goal of this work is to design and implement an easy to use distributed (swarm) robotics platform for the purposes of performing a basic task at a series of remote, geographical locations, in this case to simply take a measurement thereby performing a form of environmental bsensing. The overall system will contain an arbitrary amount of robots, with the capability that robots can enter or leave the swarm at any time. 
Geographic locations are assigned to each robot as the locations are serviced, and as robots enter or leave the network. Task information (sensor data) and system status is recorded by a base station, which acts as a central node in the swarm. The base station in this case will supervise all robots in the swarm and host information available for display by the HMI. Ease of use is emphasized from the perspective of the end user, and therefore beyond an initial setup step, the user should simply interface with a pc to control the system. Controlling the system in this case is defined as simply entering the required sample points and possibly an initial reference point. Finally, since small robots are generally used for swarm applications, all software algorithms are be implemented on a microcontroller based system.

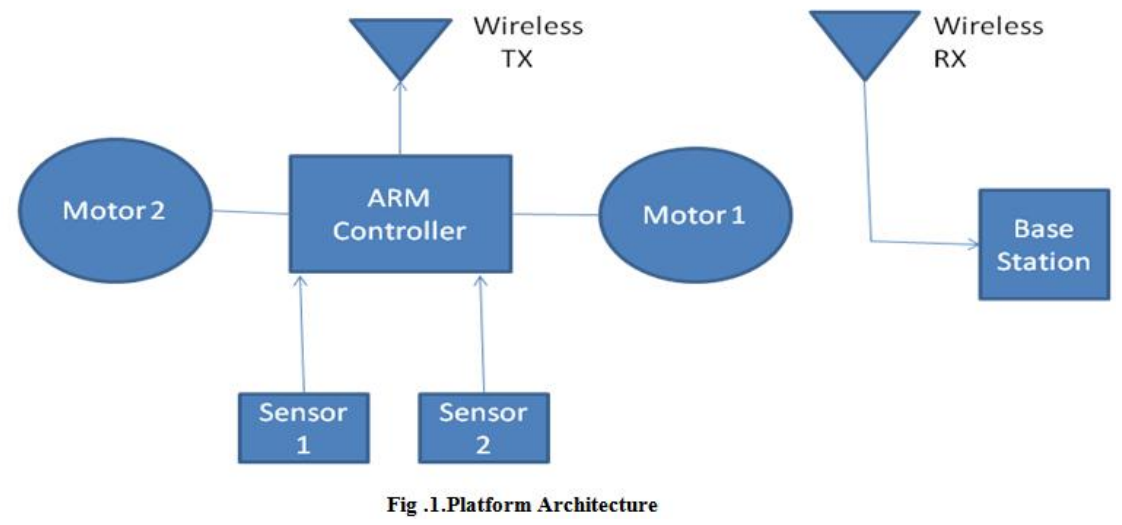

\subsection{MRDI Database}

III. Mobile Robot Data Interface

The mobile robot data interface (MRDI) provides a transparent protocol for a base station to access and mutate variables on the robot, and to issue commands where appropriate. A SCADA system, popular for automation applications, performs similar functions in a robust fashion, and thus the SCADA architecture is used for this design. ASCADA system deploys a server hosting a database of variables and commands. A $\mathrm{read} / \mathrm{write}$ to a variable or command in the database, causes the server to forward that read/write to appropriate controller module. The advantages to this system are that the client does not need to consider the internal workings of the database, or the controller module downstream being affected by the read/write. Additionally, the protocols used to facilitate this operation ensure controller modules are alive, functioning etc. Thus it is plain that the advantages of such a system include transparency of access and fault tolerance. Since the swarm robotics system is a network of controllers, much like an industrial system, following SCADA system principles intrinsically leverages its advantages in the design of the communications and data exchange component. Based on the overall system's objective, a summary of requirements is listed: microcontroller friendly database; transparent read/write support; low bandwidth communication support; and a robust communication protocol.

The MRDI component includes both the "SCADA" database and a communication protocol optimized for a RF-transreceiver network and for operations performed on the database. A copy of the database is implemented on each robot and is compiled together with the operating system and other application code. Each element of the database pertains to a relevant variable or command associated with the application of the robot (eg. Current robot location). Reads and writes to the database on a local level are handled by an API, adjusting the element's current value, or returning the current value to the calling function and executing a callback function defined by the user while creating the database. The use of a callback function allows a database element to implement a command or action which is linked with robot operation. Additionally, callback functions can be used to implement inter-task communication and interfacing to I/O drivers. Since interfacing in this regard is performed via reading and writing to tags, the data exchange is visible to the remote base station, thus aiding debugging and faultmanagement.

The MRDI database is integrated using the following steps: (1) Design robot (user) application software (Control System, Intelligence, etc.); (2) Identify data elements to be shared and remote commands; (3) Generate database using database creation tool; (3) Implement robot (user) application code using MRDI (4) Compile user code, database together for target.

\subsection{MRDI Communications}

Using the message paradigm, the MRDI communication protocol uses several different types of small messages (less than 32 bytes) to facilitate discovery of new robots in the swarm, monitoring of existing robots for communication failure (system exit), and performing read/write operations for database access. A message based approach is used, as messages can be made to fit inside a single link layer packet. Thus, link layer 
robustness features can be leveraged at the transport layer, and do not have to be implemented at this level. Robot discovery is performed by the base station periodically transmitting "discover" messages on the RFtransreceiver network using a broadcast address. All robots, including those not in the swarm will reply. The base station can then use these replies to determine if any new robots have come RSSI since the last discover message, and thus can be added to the system and assigned tasks. Heartbeat messages are periodically sent from the base station as well, to each robot in the swarm on a robot by robot basis (nonbroadcast). The robot can use these "heartbeat" messages to determine if the base station is still RSSI, and if not, it can perform an application specific action, through notification and callback of a database variable. As well, the base station uses the heartbeat messages to determine if a given robot is no longer operational. Database read/write commands from the base station are packaged into "database" messages, and processed by the communication stack as if a local $\mathrm{read} /$ write has been performed; except returned data is repackaged and transmitted back to the base station. It is important to note, that databaseelements are referenced by index. Therefore the base station must have prior knowledge to map variable names to index Total message size ( 32 bytes) is constant to ensure determinism and to easily estimate the network load for a swarm of given size. This allows future work to optimize the polling rates for "discover" and "heartbeat" messages, as well as the polling of data from each robot. Finally, multiple read and writecommands can be integrated into a single message. This ensures efficiency when a large amount of data needs to be handled in a very short period of time.

\subsection{MRDI Base Station}

The MRDI base station is a protues software module which implements the MRDI communication protocol and maintains a list of available robots in the swarm. This module then provides an API to a higher level user base station implementation. The user base station implementation, which is application specific, manages the system response for robot's entering or leaving the swarm, and controlling the specific tasks of each robot. The list of active robots in the swarm, is of course updated when a robot is no longer responding to heartbeat messages or database read/writes, or if a "discover" message is received pertaining to a robot not currently listed.

Reading and writing information to robots requires knowledge of the database as previously mentioned. A copy of each database created and active in the swarm is maintained by the base station, and is used to facilitate read/writes to a robot by mapping variable names to database indices. Reading and writing methods can then create appropriate read/write messages by mapping the required variable name to its respective index, and transmit that message to a robot active in the swarm. Robots can then be uniquely identified, if required, through their physical media access controller addresses. In the event that a robot becomes offline at any time, protues error handling facilities are used to notify the user`s implementation, such that it can be handled appropriately.

\subsection{Platform Requirements}

\section{Robotic Platform}

Since remote locations are specified, it follows that an unmanned aerial vehicle should be used as robotic platform. The robot itself is to be remotely flown to RF described coordinates, while maintaining some form of communication with a base station. Since it is assumed that the base station to robot communication connection may be of low bandwidth and/or poor quality, the robot must be able to dynamic itself to the prescribed location; otherwise a centralized method of control will not be robust in light of these assumptions, due to the potential of a lost or corrupt control signal. The ability for locally controlled autonomous swarm implies the requirement for a sensor package and control system module, which is capable of stabilizing and navigating the vehicle. Finally, both a communications system with a range of at least $15 \mathrm{KM}$ and a RF module capable of normal satellite tracking and acquisition is required to provide absolute location awareness and base station to robot communications. All of these features must integrate into a small, mediumweight package appropriate for a protable-sized swarm, which will be modified for this application. These design requirements include: autonomous swarm; communication system having $15 \mathrm{KM}$ of range; sensors and control systems capable of supporting autonomous swarm; and a RF system capable of standard satellite navigation.

\subsection{Platform Selection And Design}

To facilitate these requirements an DC-rotor type dynamic is used as a base platform. Incorporating four stepper high power motors in a very sturdy package, the platform is capable of all-weather inside swarm. Additionally, a 32 bit ARM Cortex-M4 microcontroller is used as the computational component. swarm control of the bynamic robot is facilitated using a simple PD control approach, modulating all four rotors in the unit. 


\section{Basestation And Hmi}

The base station and HMI is implemented on a pc to maximize hardware simplicity while maintaining user familiarity. For the purposes of this work, a pc was used as the target platform. The base station MRDI implementation resides on this device in order for the application and HMI software to access information from the swarm. It is important to note that the pc's wireless technologies do not include RF at this time. Therefore, a pocket sized wi-fi to RF Bridge was rapidly implemented using a wi-fi and RF module, by linking the common user side interfaces (UART) together in a crossover fashion. This bridge is pocket sized and can be carried on the user's person at no extra burden. The application code on the pe utilizes the MRDI stack to continuously poll robots in the swarm for relevant information, and, in the case of the environmental sensing problem, assign a geographic location to each robot, as locations are serviced in turn. Polling of data from each robot is handled on a periodic basis, definedby a "poll list", which is simply a list of database variables which must be continuously read for application and HMI purposes.

The HMI, integrated with the PC native maps application, displays the location of each robot's position in, overlaid on a map of the relevant locale.Additionally, relevant operational status metrics of each robot, and application information is presented to the user. The exact look and feel of the HMI, as well as the information presented is highly application dependent. However, based on the open nature of the MRDI stack and smartphone application development, the HMI can easily be tailored to meet application requirements.

\section{Conclusion And Future Work}

The operation of the operating system, MRDI stack, and HMI at a basic level, indicates the viability of the whole system to be used for other swarm robot applications. Since the entire platform is a composed of distinct components, each component can be separated and used for a variety of different applications. Additionally, the ability for the system to be reconfigured for any robot platform, allows itbe used as a useful research tool by allowing the researcher to focus on application level components, instead of low level infrastructure integration. Finally, since this work involves a large number of sub-components, researchprojects can be built around each of these sub-components.Future work will include polishing the HMI component, and completing the swarm platform's control system.Following completion of this work, it is expected that the entire platform be made close source for other to use and build upon.

\section{References}

[1] R.J. Mullen, S. Barman, P. Remagnino, and D. Monekosso, "Towards autonomous robot swarms for multitarget localisation and monitoring with applications to counter ied operations," International Journal of IntelligentDefence Support Systems, vol. 4, no. 1, pp. $87-107,2011$

[2] F. Viani, M. Donelli, G. Oliveri, and A. Massa, "A mobile wireless sensor network architecture for collaborative tasks achievement by means of autonomous robot swarm," 2010 IEEE International Symposium on Antennas and Propagation and CNC-USNC Radio Science Meeting - Leading the Wave, AP-S 2010, 2010

[3] C. Vasile, A. Pavel, and C. Buiu, "Integrating human swarm interaction in a distributed robotic control system," 2011 IEEE International Conference on Automation Science and Engineering, pp. 743 - 748, 2011.

[4] S.H. Kenyon, D. Creary, D. Thi, and J. Maynard, "A small, cheap, and portable reconnaissance robot," Proceedings of SPIE - The International Society for Optical Engineering, vol. 5778, no. PART I, pp. 434 - 443, 2005.

[5] K. Daniel, B. Dusza, A. Lewandowski, and C. Wietfeld, "Airshield: a system-of-systems muav remote sensing architecture for disaster response," 2009 IEEE International Systems Conference, pp. 5 pp. -, 2009.

[6] S.M. Cameron, G.M. Loubriel, III Robinett, R.D., K.M. Stantz, M.W. Trahan, and J.S. Wagner, "Adaptive remote sensing techniques implementing swarms of mobile agents," Proceedings of the SPIE - The International Society for Optical Engineering, vol. 3713 , pp. $160-177,1999$.

[7] S. Haldar and D.K. Subramanian, "A modified round robin scheduler for time-sharing systems," Computer Journal, vol. 35, no. 3, pp. $309-315,1992 / 06$. 